\title{
Use of CTFFR and CTCA to Diagnose and Triage Patients of INOCA: A Retrospective Study
}

\author{
Atul Kapoor, Goldaa Mahajan, Aprajita Kapur \\ Department of Radiodiagnosis, Advanced Diagnostics and Institute of Imaging, Punjab, India
}

Email address:

masatulak@aim.com (A. Kapoor)

\section{To cite this article:}

Atul Kapoor, Goldaa Mahajan, Aprajita Kapur. Use of CTFFr and CTCA to Diagnose and Triage Patients of INOCA: A Retrospective Study. Cardiology and Cardiovascular Research. Vol. 5, No. 2, 2021, pp. 67-73. doi: 10.11648/j.ccr.20210502.13

Received: April 18, 2021; Accepted: May 7, 2021; Published: May 14, 2021

\begin{abstract}
Objectives: INOCA(Ischemia in non- obstructive coronary arteries) has been recognized as a global health problem and poses a diagnostic challenge to establish the diagnosis which involves first ruling out obstructive coronary artery disease by the use of CT coronary angiography (CTCA) or an invasive angiogram. Second step involves the use of intracoronary pressure and Doppler monitoring which is not only costly, time consuming and lacks easy availability. CTFFR has emerged as frontline tool in the non invasive evaluation of patients with stable chest pain. This retrospective study was designed to evaluate the spectrum of findings of ischemia on CTCA and CT FFR in patients with stable chest pain to determine if this protocol can be used to identify patients with INOCA before they are subjected to invasive protocol. Methods: This was a retrospective study of 500 consecutive patients of stable chest pain with more than $>1 \mathrm{~mm}$ ST depression on resting EKG and or positive stress test who underwent CTCA along with CTFFR evaluation using a prescribed CT angiographic protocol. Post processing was done to reconstruct multiplanar angiographic views followed by CT FFR evaluation. All patients with no obstruction or stenosis less than $50 \%$ and with CT FFR of $<0.80$ were labeled as having INOCA. Subcategorisation of all INOCA patients was done based on Vessel tapering index (VTI), Plaque volume Index (PVI) into four subtypes- TypeI (vasospastic), Type II (site specific atherosclerotic), Type III (distal macrovascular dysfunction- DMD), Type IV (mixed). Results: Study showed 122 (34\%) patients of stable chest pain had INOCA. Types I, III formed the largest group of patients $38 \%$ and $31 \%$ followed by the other two subtypes and showed significant differences in the VTI and PVI along with reduced FFR of $<.80$ in all these patients. Conclusion: Use of CTCA and CTFFR can be used as a first line tool to not only rule out obstructive coronary disease with ischemia but also to non invasively detect INOCA in patients with stable chest pain before subjecting these patients for further invasive protocols and can influence accurate management of such patients.
\end{abstract}

Keywords: INOCA, CTFFR, CT Coronary Angiography

\section{Introduction}

INOCA (ischemia in non-obstructive coronary arteries) has been recognized as a major health problem globally. $40 \%$ of the patients of stable chest pain who undergo coronary angiography have normal coronary arteries and despite normal angiograms have ischemia with non-obstructive coronary disease i.e. INOCA [1]. Patients presenting with this syndrome have symptoms and signs suggestive of ischemic heart disease but are found to have non obstructed coronary arteries [2]. These patients frequently are managed inappropriately as non-cardiac and hence continue to remain symptomatic. Many of these patients with INOCA also have coronary micro vascular dysfunction (CMD) and are at a higher risk of major adverse cardiac events. Diagnosis for INOCA thus requires invasive testing with the use of pressure wire insertion, intracoronary drug injections and use of Doppler monitoring as per ECP guidelines. WISE trial [3] showed that 1 in 3 women with non- obstructive ischemia died in a ten year follow up. Therefore a disease which was earlier considered benign did not have any recommended treatment guidelines needs careful diagnosis and management. Recently consensus guidelines have been issued by European Society of cardiology [4] regarding evaluation and diagnosis of suspected patients of INOCA in which CTCA has been included as the mainstay investigation to rule out obstructive coronary artery disease. This is followed by invasive evaluation using intracoronary acetylcholine and adenosine to 
establish the diagnosis $[5,6]$. Considerable evidence now documents that this syndrome is associated with a prognosis that is clearly not benign, yet no clinical practice management guidelines exist for these patients. There is also likely overlap between INOCA and myocardial infarction with no obstructive coronary arteries (MINOCA) and heart failure with preserved ejection fraction again indicative of a nonbenign nature of the problem requiring a careful diagnosis [7].

FFRCT has recently emerged as frontline procedure combined with CT coronary angiography (CTCA) for non-invasive testing as per NICE guidelines 2019 in patients with stable chest pain [8]. This algorithm has so far been used to identify obstructive coronary artery disease with significant ischemia distal to the culprit lesion to plan percutaneous intervention and management. The two most common causes of INOCA -microvascular angina (MVA) and vasospastic angina (VSA) are also neither excluded by a negative non-invasive CT coronary angiogram nor invasive coronary angiogram. As cardiologists, we have so far adopted a 'stenosis-centric' approach [9] and missed this identity by our routing diagnostic protocols.

Recently Lin et al [10] have shown that the use of CTFFR can detect co localized reduced FFR in non-obstructive vessels with high plaque burden and these were easily missed by invasive FFR. Similarly in CorCTCA trial [11] the role of CTCA in the diagnosis of INOCA is being studied.

We retrospectively reviewed the clinical and imaging data of 500 patients of stable chest pain who underwent CTCA and identified patients having INOCA with no significant stenosis but with reduced CTFFR $<0.80$. These patients were phenotyped into categories based on the pathological features of the vessels studied. To our knowledge this is the first study which identifies patients of INOCA non-invasively and phenotypes the disease to plan further management.

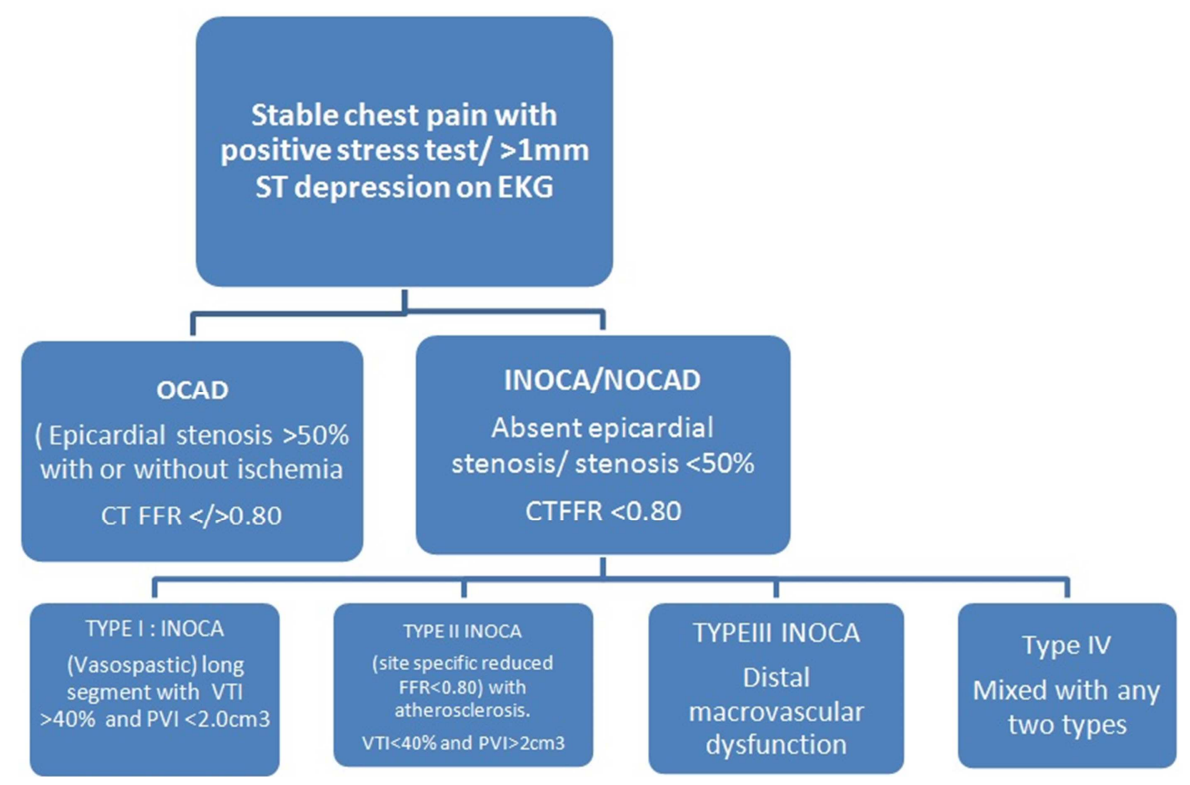

Figure 1. Flow chart showing evaluation of patients with stable chest pain.

\section{Material and Methods}

This is a retrospective study comprising of 350/500 consecutive patients of stable chest pain with more than $1 \mathrm{~mm}$ ST segment changes on electrocardiogram or positive stress tests. The demographic parameters of all patients i.e. age, sex, BMI alongwith history of disease, coexisting morbidities like hypertension, diabetes and hyperlipidemias were recorded. All patients with history of allergy to iodine, arrhythmias, heart rate of more than 100 beats $/ \mathrm{mt}$, BMI more than $31 \mathrm{Kg} / \mathrm{m}^{2}$, increased creatinine levels $>1.2 \mathrm{mg} / \mathrm{ml}$. After informed consent all patients underwent coronary computed tomographic angiography (CTCA) on a dual energy CT system (Siemens Go-Top) 128 slice scanner after receiving oral $50 \mathrm{mg}$ metoprolol one hour before the examination. Tube voltage was set according to the group of patients being done using the protocol described. A retrospectively gated ECG triggered spiral (pitch 0.3) acquistion was done with collimation of and gantry rotation time of $0.31 \mathrm{~ms}$ in both the groups. The tube voltage was $70-80 \mathrm{KV}$ along with automated tube current modulation using (CARE Dose 4D, Siemens healthineers) and the quality reference voltage and current was set to $120 \mathrm{kV}, 320 \mathrm{mAs}$. R-R interval was fixed at $35-75 \%$. $35 \mathrm{ml}$ of non iodinated contrast iomperol 400 (Iomeron Bracco UK Ltd) was injected using bolus tracking soft ware with dual head injector (MEDRAD, Stellant, Bayers, Munich, Germany) with flow rate of $4 \mathrm{ml} / \mathrm{s}$ followed by $20 \mathrm{ml}$ saline. Image reconstruction was done using iterative reconstruction (SAFIRE, Siemens healthineers level 3) with reconstruction kernel of BV36. All images were transferred to Siemens Syngo. Via workstation for post processing in multiplanar and volume rendering projections with calculation of vessel tapering index (VTI) Proximal vessel diameter - Mid vessel diameter/proximal vessel diameter $\times 100 \%$. CTFFR was done onsite on Siemens Syngovia Frontier workstation using CTFFR (version 2.0) software. CTFFR was displayed in the form of color maps of coronary tree with blue being normal and red being ischemic (i.e. FFR $<0.80$ ). Plaque volume index 
(PVI) i.e., plaque volume $(\mathrm{cm} 3) /$ segment length of vessel $\mathrm{x} 100 \%$, type of plaque fibrous, lipid, mixed or calcified was calculated using Plaque analysis software on Siemens Syngo Frontier workstation. All patients with reduced CTFFR of $<0.80$ were labeled as having ischemia and were categorized into Obstructive and Non obstructive coronary artery disease as per flow Figure 1. Patients with non-obstructive coronary artery disease (INOCA) were classified into four phenotypes. Type 1: having vasospastic type of vessels with VTI of $>40 \%$ and PVI of $<2.0$. Type 2: Atherosclerotic-site specific type with VTI of $<40 \%$ and PVI of $>2.0$. with stenosis less than 50\%. Type3: Distal or branch type. Type 4: mixed type with any two of above. All patients with coexisting obstructive coronary artery disease were excluded.

\section{Results}

Study shows that $122 / 350$ (34\%) patients with stable chest pain had evidence of INOCA on CTFFR. Out of these 52\% were females in type I group who had a vasospastic phenotype while other three groups showed no difference in sex predilection. The Mean age group of patients in all the four groups was $55.5+-2.5$ years. Other patient demographics are shown in Table 1. Type I (vasospastic group) formed the largest number of patients 47/122 (38\%) and showed a higher VTI of $44.5 \%$ while other groups showed a VTI of less than $20 \%$ the difference being statistically significant $(\mathrm{p}<0.01)$. The mean PVI was 1.8 in this group (Figures 2-4). Group III i.e., with distal macrovascular endothelial dysfunction (DMD) was the second largest group 38/122 (31\%) (Figures 5-7) and showed a normal TVI with mean PVI of $2.4 \%$ with 11 patients showing DMD in branch vessels. Group II patients were those with atherosclerotic site-specific phenotype of INOCA and were $21 / 122(17.2 \%)$ in number and showed a normal TVI of $18 \%$ with a higher PVI of $3.5 \%$. The mean vessel stenosis in this group of $32 \%$ (Figures $8-10$ ). Group IV was those with mixed type I, II, III phenotypes and had 16/122 (13\%) patients and showed TVI of $22 \%$ with PVI of $2.8 \%$ (Figures $11-13$ ).

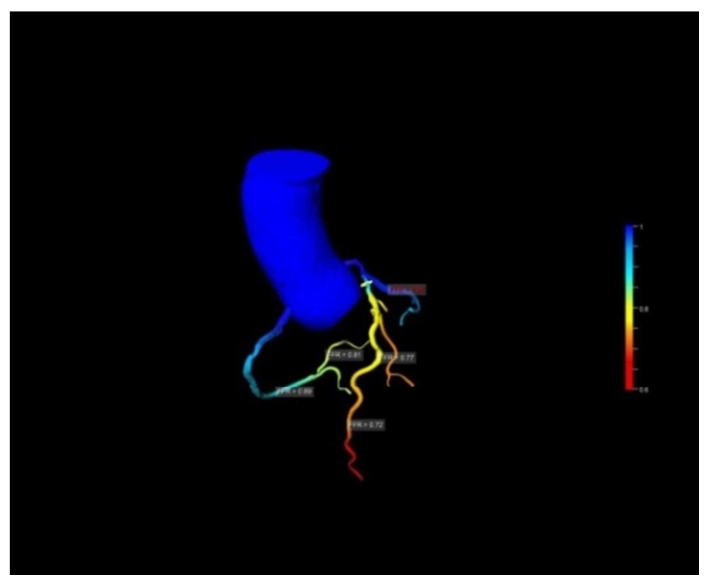

Figure 2. CTFFR group I patient showing reduced FFR of 0.77 and 0.72 in mid and distal left anterior descending artery.

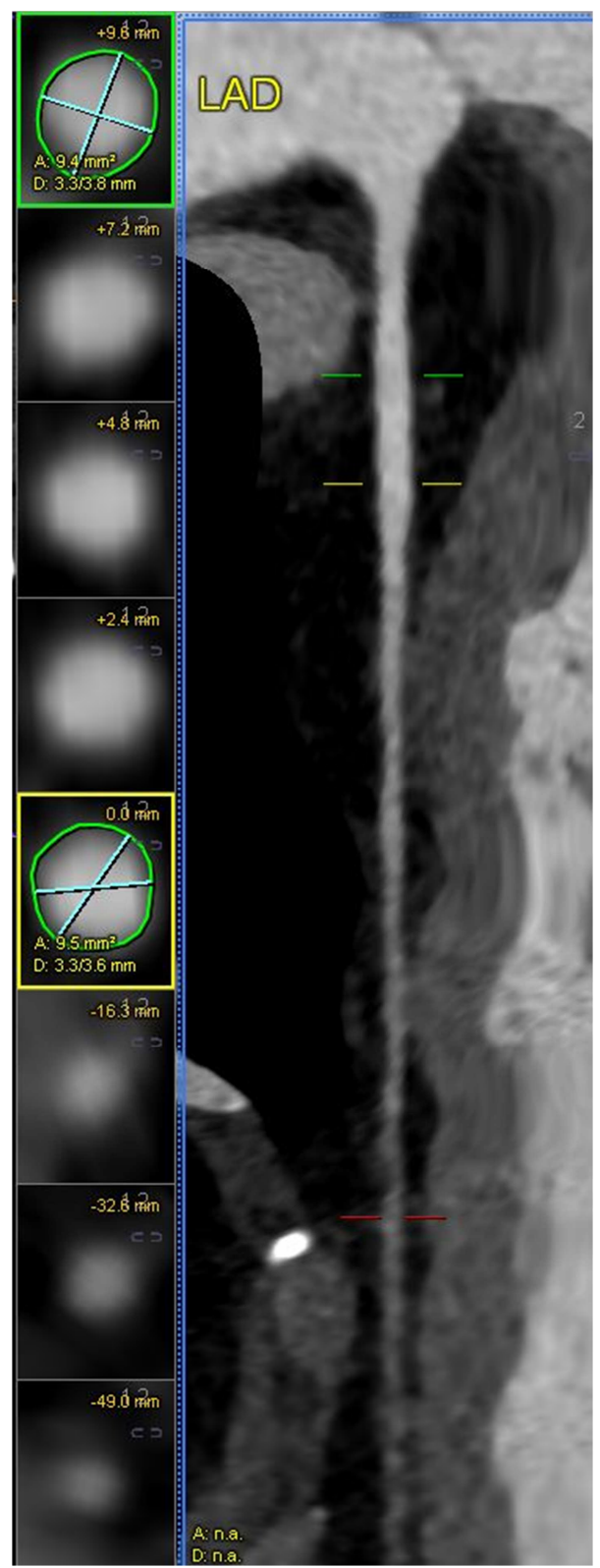

Figure 3. CTCA straightened multiplanar reconstruction image of left anterior descending artery with VTI of $55 \%$.

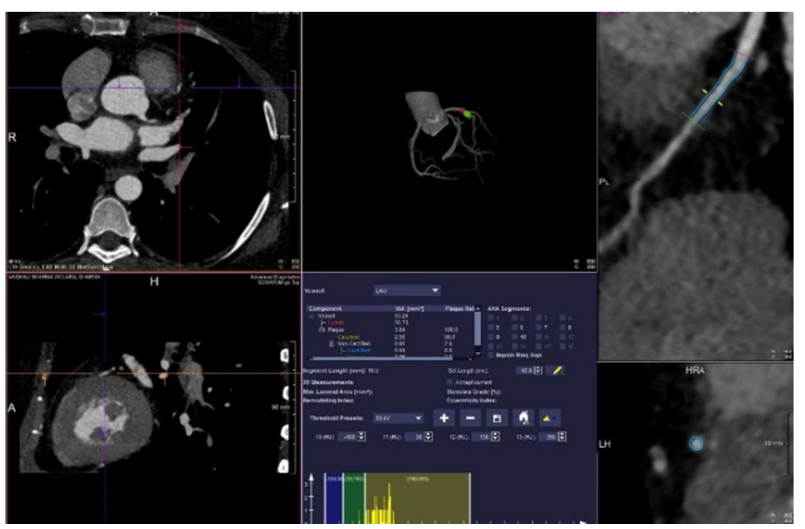

Figure 4. Plaque analysis of left anterior descending artery showing PVI of less 2.0. 


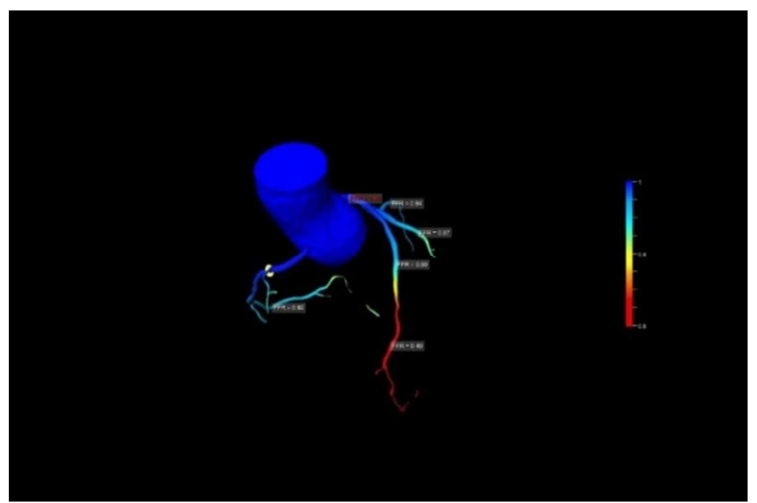

Figure 5. CTFFR of group III patient showing distal reduced FFR of 0.67 (DMD type) in distal Lt anterior descending artery.

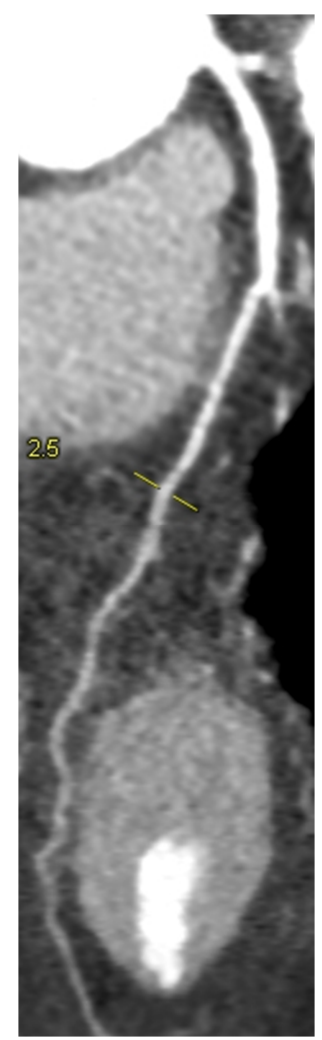

Figure 6. CTCA of same patient showing VTI of $12 \%$.

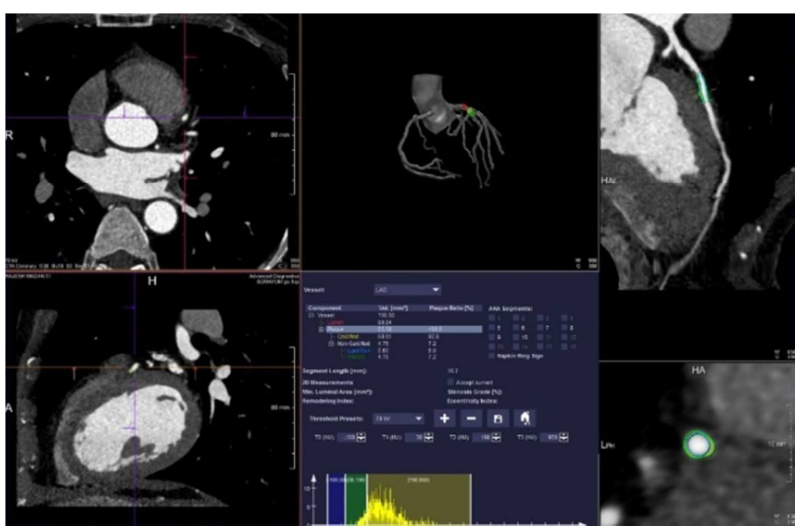

Figure 7. Plaque analysis showing PVI of $3.5 \%$ in the proximal segment of left anterior descending artery.

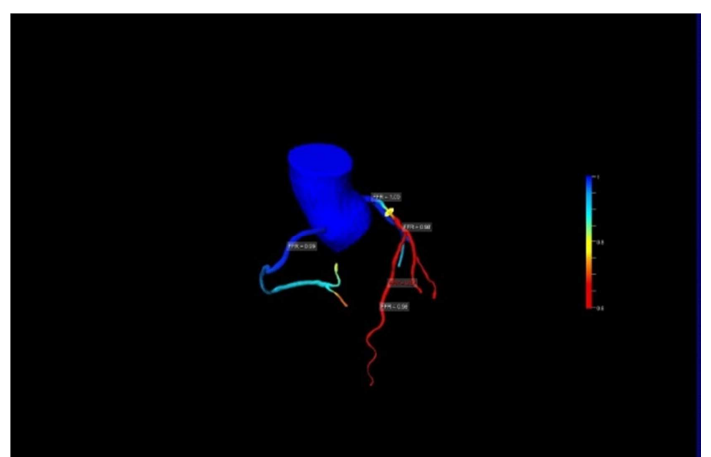

Figure 8. Group II patient with site specific reduced distal FFR of 0.58 in left anterior descending coronary artery.

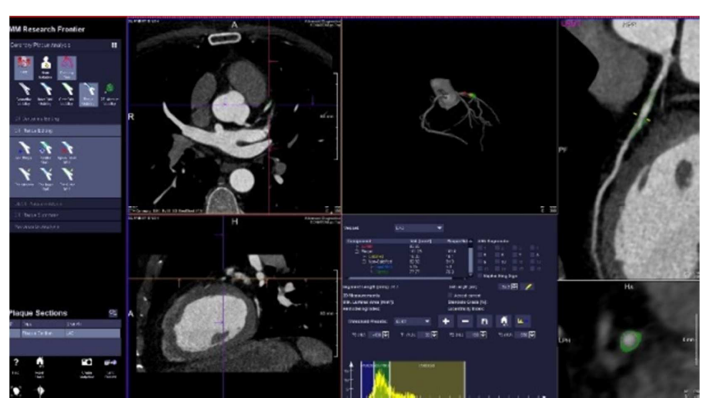

Figure 9. Plaque analysis same patient showing PVI of $3.8 \%$ in left anterior descending coronary artery.

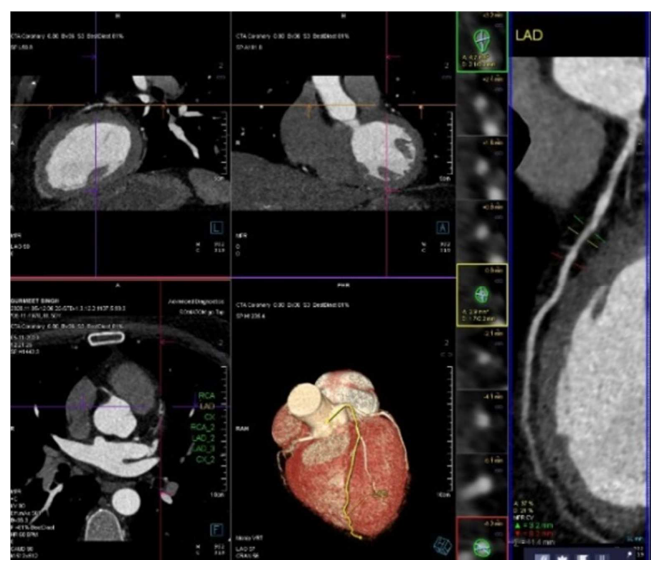

Figure 10. CTCA showing mixed plaque in proximal left anterior descending artery with VTI $17 \%$ with mild stenosis of $35-37 \%$.

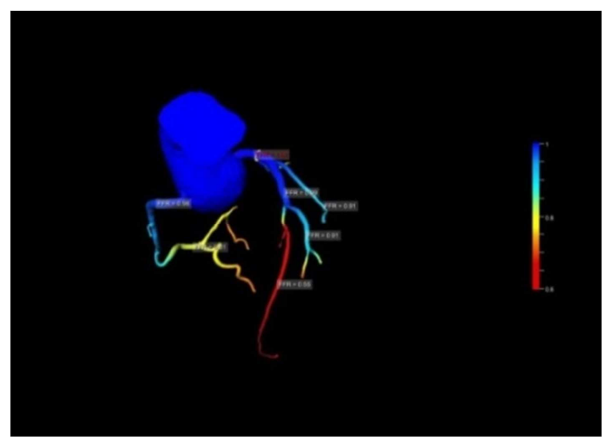

Figure 11. CTFFR group IV patient with reduced FFR of 0.55 in mid and distal Left anterior descending artery-VSA type and distal right coronary artery-DMD type. 


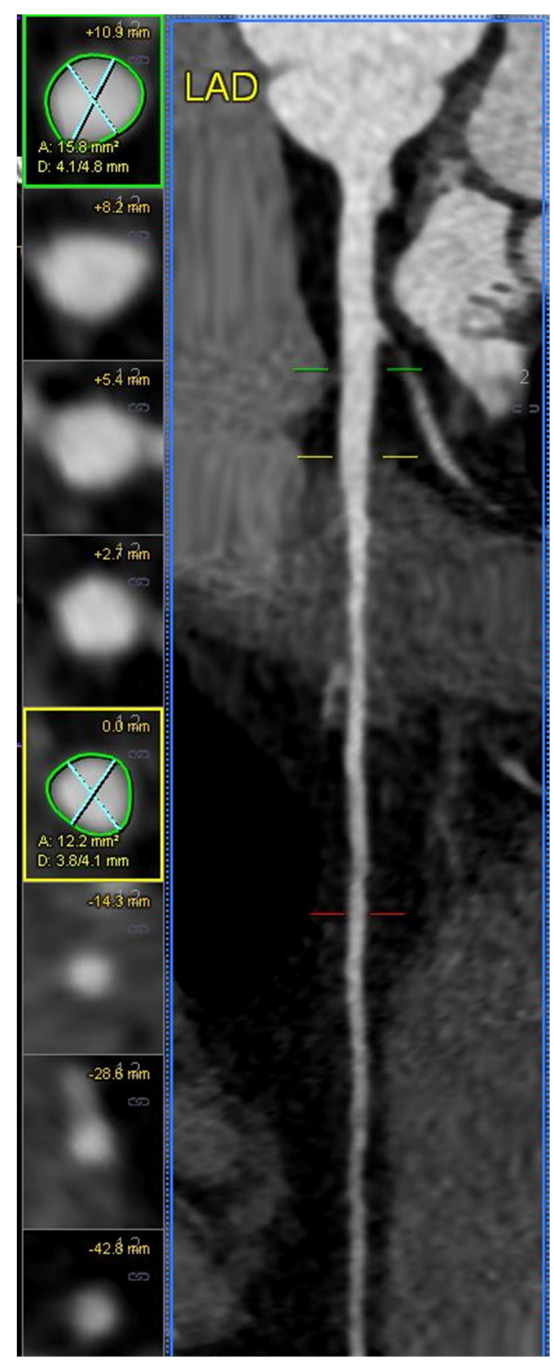

Figure 12. CTCA image of the Left anterior descending artery showing VTI of $45 \%$ in mid segment.

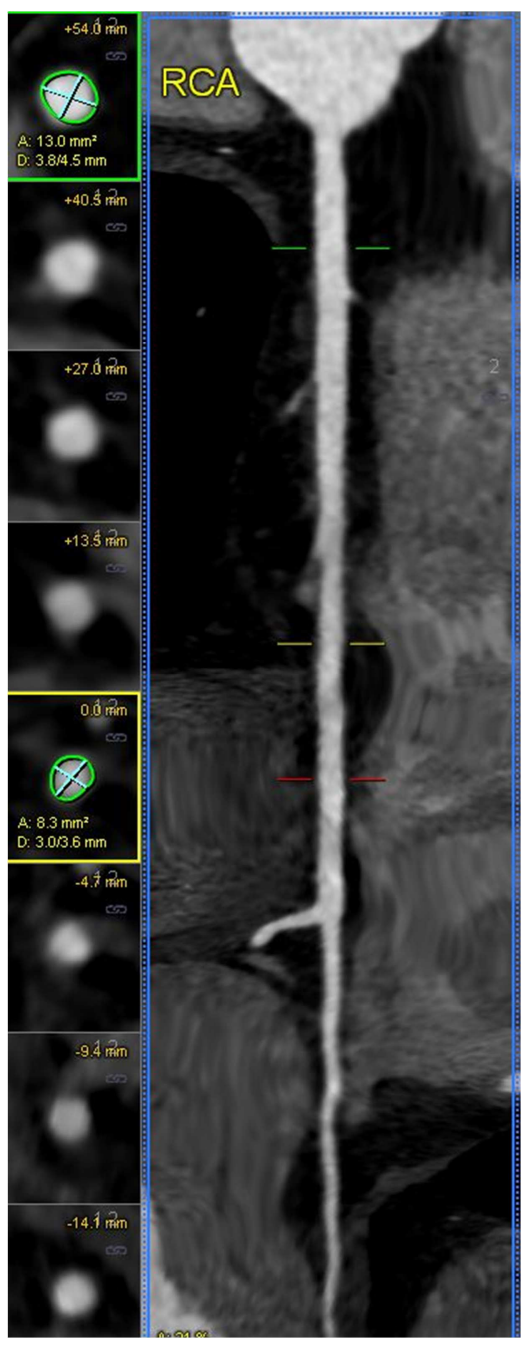

Figure 13. CTCA image of right coronary artery with VTI of $11 \%$ in mid right coronary artery.

Table 1. Patient Demographics of INOCA Phenotype groups.

\begin{tabular}{llllll}
\hline & & Type I & Type II & Type III & Type IV \\
\hline S. No. & Variable & $\mathrm{n}=45$ & $\mathrm{n}=21$ & $\mathrm{n}=38$ & $\mathrm{n}=18$ \\
1 & Mean Age & 53.3 & 55.5 & 52.5 & 51 \\
2 & SEX & & & 51 & 55 \\
& Males & 48 & 55 & 49 & 45 \\
\\
3 & Females & 52 & 45 & 28 & 29.1 \\
4 & BMI (KG/M2) & 30 & 28.5 & 65 & 62 \\
5 & Hypertension & 38 & 72 & 28 & 0 \\
6 & Diabetes & 18 & 32 & 1 & 48 \\
7 & Smoking & 5 & 55 & 4 & 6 \\
8 & Dyslipidemia & 33 & 6 & & \\
& Family History & 8 & & & \\
\hline
\end{tabular}

Table 2. Vessel and plaque morphology in INOCA Phenotypes.

\begin{tabular}{lllll}
\hline & Type I & Type II & Type III & Type IV \\
\hline Tapering index (VTI) \% & 44.5 & 15 & 18 & 18.5 \\
Plaque types & & & $60 \%$ & $45 \%$ \\
Soft & $65 \%$ & $45 \%$ & 20 & 35 \\
Mixed & $20 \%$ & $10 \%$ & 20 & 20 \\
Calcified & $15 \%$ & $45 \%$ & 2.4 & 3.2 \\
Plaque volume index (PVI)\% & 1.80 & 3.1 & $20 \%$ & $25 \%$ \\
Coexisting Stenosis & $0 \%$ & $5 \%$ & & \\
\hline
\end{tabular}




\section{Discussion}

The present study had $33 \%$ patients with stable chest pain on CTCA with electrocardiogram findings of ischemia and had INOCA detected with the use of CT FFR. All these patients were falsely labeled on CTCA alone as having no significant coronary artery disease and continued to be wrongly treated due to missed diagnosis. Similar results were shown by COR MICA trial [12] in which 50 percent of the patient's treatment was changed after the results of functional coronary testing after normal angiograms. This leads to improved quality of life and angina control. Similarly WISE trial also showed $62 \%$ of women referred for coronary angiography did not have obstructive coronary disease but had non obstructive ischemia [3]. There have been practical problems in not only choosing patients of stable chest pain with normal angiograms for further functional invasive testing as suggested by European ESC guidelines but also availability of these pressure monitoring techniques along with the cost have also been deterring factors as so for. Further the guidelines from the Coronary Vasomotion Disorders International Study (COVADIS) Group which mandate invasive coronary reactivity testing to establish a definitive diagnosis of INOCA Coronary reactivity testing have shown a diagnostic yield of only $60 \%$ in identifying vasospasm and coronary macrovascular dysfunction with additional procedural risks up to $0.7 \%$ [13]. Current study shows that the use of CTFFR with CTCA in these patients can not only diagnose ischemia non-invasively but also can be phenotyped into four subtypes categories which can form the basis for further invasive testing to confirm the diagnosis. This being a retrospective study invasive testing was not performed. Findings of current study suggest a tiered approach to screen and diagnose more patients of INOCA who otherwise are missed based on the findings of angiography alone as has been suggested by The Coronary Microvascular Angina randomised-controlled trial (CorMicA) who recently demonstrated a similar approach which was better than the usual care [12]. Our study shows that phenotype I (vasospastic) patients formed the largest group of patients of INOCA with a high tapering index of vessels compared to other phenotypes and was likely due to reduced nitrous oxide levels in these patients [14]. We feel that the use of sublingual nitrate while conducting CTCA helps to unmask the underlying vasospastic condition of the vessel. Normal coronary arteries show a VTI of $18-20 \%$. Estimation of vessel tapering index of more than $40 \%$ was along with reduced FFR was a good non-invasive parameter to diagnose this phenotype as shown in this study. The study also shows that this group of patients had very little plaque burden with PVI $<2.5 \mathrm{~mm}^{2}$. Type III group formed the second largest phenotype of patients who had distal macrovascular dysfunction (DMD) which was a subtype of coronary microvascular angina. Patients with DMD have been shown to have reduced CFR or microvascular dysfunction by coronary invasive flow testing. Detection of this phenotype also has prognostic importance as this has been shown by
DEFINE Flow trial [15] in which patients with reduced FFR and CFR i.e., microvascular dysfunction fared better post PCI than those with reduced FFR but with normal CFR. There were equal number of patients in this study with type II and type IV phenotypes which were having atherosclerotic and mixed phenotypic appearances and showed no sex predilection. PVI in these two groups was high i.e., $>4.5 \mathrm{~mm}^{2}$ and there was predominant fibrotic type of plaque morphology and the reduction of FFR was site specific in all these patients with reduction of lumen diameters being less than $50 \%$. Lin et al [10] recently also showed similar results and identified 58 /71 patients of INOCA on FFRCT who underwent myocardial perfusion imaging and invasive FFR. In their study there was a high prevalence of macrovascular coronary plaque and abnormal co-localized FFRCT. Only 35\% of these lesions were detected on invasive FFR studies. They suggested that distal reduced FFR and the non-site specific reduced FFR's were missed by invasive FFR without plaque localization. Their study concluded FFRCT could detect INOCA especially in patients with high-risk plaque features or those with small vessels and these could be missed by invasive techniques.

\section{Conclusion}

Our study concludes that the use of CTFFR along with CTCA in the diagnostic protocol of a patient with stable chest pain as per NICE 2019 guidelines not only rules out obstructive coronary artery disease but can be a gateway to detect and triage patients having INOCA for invasive coronary testing. Such a diagnosis can not only lead to early and accurate diagnosis but also alter the patient management and prognosis including long term high mortality. The current study for the first time highlights the imaging phenotypes of INOCA by the use of FFRCT and CTCA which can has diagnostic and therapeutic implications.

This study being a retrospective study has limitations that no coronary reactive testing or any perfusion study was done to further evaluate the diagnosis.

\section{Acknowledgements}

Siemens Healthineers, Erlangen Germany, Syngovia Group, Bangalore, India.

\section{References}

[1] GBD 2015 Mortality and Causes of Death Collaborators. Global, regional, and national life expectancy, all-cause mortality, and cause-specific mortality for 249 causes of death, 1980-2015: a systematic analysis for the GlobalBurden of Disease Study 2015. Lancet. 2016; 388: 1459-1544.

[2] Reeh J, Therming CB, Heitmann M, Hojberg S, Sorum C, Bech J, Husum D, Dominguez H, Sehestedt T, Hermann T, Hansen KW, Simonsen L, Galatius S, Prescott E. Prediction of obstructive coronary artery disease and prognosis in patients with suspected stable angina. Eur Heart J. 2019; 40: 1426-1435. 
[3] Johnson BD, Shaw LJ, Pepine CJ, Reis SE, Kelsey SF, Sopko G, Rogers WJ, Mankad S, Sharaf BL, Bittner V, Bairey Merz $\mathrm{CN}$. Persistent chest pain predicts cardiovascular events in women without obstructive coronary artery disease: results from the NIH-NHLBI-sponsored Women's Ischaemia Syndrome Evaluation (WISE) study. Eur Heart J. 2005; 27: 1408-1415.

[4] Kunadian V, Chieffo A, Camici PG, Berry C, Escaned J, Maas $\mathrm{AH}$, Prescott $\mathrm{E}$ et al. An EAPCI Expert Consensus Document on Ischaemia with Non-Obstructive Coronary Arteries in Collaboration with European Society of Cardiology Working Group on Coronary Pathophysiology \& Microcirculation Endorsed by Coronary Vasomotor Disorders International Study Group European Heart Journal (2020) 41, 3504-3520.

[5] Camici PG, Crea F. Coronary microvascular dysfunction. $N$ Engl J Med. 2007; 356: 830-840.

[6] Widmer RJ, Samuels B, Samady H, Price MJ, Jeremias A, Anderson RD, Jaffer FA, Escaned J, Davies J, Prasad M, Grines C, Lerman A. The functional assessment of patients with non-obstructive coronary artery disease: expert review from an international microcirculation working group. EuroIntervention. 2019; 14: 1694-1702.

[7] Agewall S, Beltrame JF, Reynolds HR, Niessner A, Rosano G, Caforio AL, De Caterina R, Zimarino M, Roffi M, Kjeldsen K, Atar D, Kaski JC, Sechtem U, Tornvall P, WG on Cardiovascular Pharmacotherapy. ESC working group position paper on myocardial infarction with non-obstructive coronary arteries. Eur Heart J. 2017 Jan 14; 38 (3): 143-153.

[8] Kelion AD, Nicol ED. The rationale for the Primacy of coronary $\mathrm{CT}$ angiography in the National Institute for health and care excellence (NICE) guideline (CG95) for the investigation of chest pain of recent onset. J Cardiovasc Comput Tomogr 2018; 12: 516-22.
[9] Tavella R, Cutri N, Tucker G, et al. Natural history of patients with insignificant coronary artery disease. Eur Heart J Qual Care Clin Outcomes 2016; 2: 117-24.

[10] Lin F, Gianni U, Lu Y, Stuijfzand, Bax AM, Hollenberg EJ. Macrovascular abnormalities and FFRCT in ISCHEMIA with non obstructive coronary arteries (INOCA): The Credence Trial. J Am CollCardiol. 2020 Mar, 75 (11_Supplement_1) 22.

[11] Sidik NP, McEntegart M, Roditi G, Ford TJ, McDermott M, Morrow A, Byrne J, Adams J, Hargreaves A, Oldroyd $K G$, Stobo D, Wu O, Messow CM, McConnachie A, Berry C. Rationale and design of the British Heart Foundation (BHF) Coronary Microvascular Function and CT Coronary Angiogram (CorCTCA) study. Am Heart J. 2020; 221: 48-59.

[12] Ford TJ, Stanley B, Good R, et al. Stratified Medical Therapy Using Invasive Coronary Function Testing in Angina: The CorMicA Trial. J Am Coll Cardiol. 2018; 72 (23 Pt A): 2 841-2855.

[13] Beltrame JF, Crea F, Kaski JC, Ogawa H, Ong P, Sechtem U, Shimokawa H, Bairey Merz CN; Coronary Vasomotion Disorders International Study Group (COVADIS). International standardization of diagnostic criteria for vasospastic angina. Eur Heart J 2015; 38: 2565-2568.

[14] Lanza GA, Careri G, Crea F. Mechanisms of coronary artery spasm. Circulation 2011; 124: 1774-1782.

[15] Stegehius V, Wijntjens, VandeHoef TP, Casadonte L, Kirkeeide RL et al. Distal Evaluation of Functionalperformance with Intravascular sensors to assess the Narrowing Effect-combined pressure and Doppler FLOW velocity measurements (DEFINE-FLOW) trial: Rationale and trial design. American Heart Journal. 2020; 222: 139-145. 